\title{
Interventional Radiology (IR) Procedures: Changing perspective in view of COVID 19 in India
}

\section{Pankaj Sharma}

Department of Radiodiagnosis, AIIMS Rishikesh 249203, India

*Corresponding author: Pankaj Sharma, Department of Radiodiagnosis, AIIMS Rishikesh 249203, India

Received date: 11 September 2020; Accepted date: 16 September 2020; Published date: 21 September 2020

Citation: Sharma P (2020) Interventional Radiology (IR) Procedures: Changing perspective in view of COVID 19 in India. J Comm Med Pub Health Rep 1(3): https://doi.org/10.38207/jcmphr100239

Copyright: (C) Sharma P 2020. This is an open-access article distributed under the terms of the Creative Commons Attribution License, which permits unrestricted use, distribution, and reproduction in any medium, provided the original author and source are credited.

Almost eight months have passed in 2020 and we are yet to reach COVID 19 peak in India. In India, already forty-five lakh people are COVID positive, with more than ninety-six thousand deaths. IR Departments have changed their practice pattern, with the formation of new Institutional Guidelines, in collaboration with the Infection Control team of Hospital. A major shift in practice pattern is the formation of two teams, each comprising of Interventional Radiologist, Nurse, Technician, and other support staff. One team stays at home and the other team works in the hospital, and both teams rotate on a weekly basis. All patients are screened at the entrance of the hospital and any patient with fever or respiratory complaint or travel history from the COVID containment area needs to be tested for COVID 19. Any non-emergency procedure like Uterine artery embolization or Fallopian tube recanalization needs to be postponed at least for the next two to three months. Emergency, life- saving procedures like Gastrointestinal bleed or Renal bleed needs to be managed urgently, with all protective gear including surgical cap, face shield or google, N95 mask, donning of Personal Protective Equipment (PPE) kit including full length, long sleeve gown, and gloves. THE entire IR team should be trained about hand hygiene, donning, and doffing of PPE. If the facility has more than one IR lab, then one lab should be dedicatedly used for COVID positive or
COVID suspect patients. If only one IR lab is available, then IR procedures for COVID positive or COVID suspect patients should be scheduled at the end of the day, except for Emergency life-saving procedures. Especially, precautions should be taken for procedures in which aerosols are generated due to coughing, like Bronchial artery embolization, pleural drainage, lung biopsy, and lung ablation. IR procedures should be spaced, with sufficient time available in between, to clean the IR lab. The entry of vendors should be strictly prohibited in the IR Department. The entire staff must be motivated regularly in this stressful time, and psychological counselling must be arranged for staff, who are not able to handle this stressful time. Any staff member having fever or respiratory illness must be managed, as per Institute guidelines. The daily meeting must be conducted, as the situation can change rapidly in the COVID scenario, and the decision must be taken quickly, how to handle the emergent situation. Work from home IR team must be available on phone and on a video call, as they can be called anytime if the primary treating team gets infected by COVID positive patient. IR patient number has drastically reduced in this COVID scenario. But we must deal with this situation tactfully and change our perspective, especially our practice pattern for IR in India. 